\title{
Lutetium Lu 177-DOTA-EB-TATE
}

National Cancer Institute

\section{Source}

National Cancer Institute. Lutetium Lu 177-DOTA-EB-TATE. NCI Thesaurus. Code C154549.

A radioconjug ate consisting of Evans blue (EB) modified, tyrosine-containing somatostatin analog, Tyr3-octreotate (TATE), conjug ated with the bifunctional, macrocyclic chelating agent tetra-azacyclododecane tetraacetic acid (DOTA), and radiolabeled with the beta-emitting radioisotope lutetium Lu 177, with potential imag ing and antineoplastic activities. Upon intravenous administration, Lutetium Lu 177-DOTAEB-TATE binds to somatostatin receptors (SSTRs), with high affinity to type 2 SST Rs (SSTR2s), present on the cell membranes of many neuroendocrine tumor (NET) cells. Upon binding and internalization, this radioconjugate specifically delivers a cytotoxic dose of beta radiation to SST R2-positive cells. The incorporation of an albumin-binding moiety through EB modification allows Lutetium Lu 177-DOTA-EB-TATE to reversibly bind to endogenous albumin, potentially extending half-life and increasing targeted accumulation of the drug in tumors. SSTRs, especially SSTR2s, are expressed at relatively higher levels in many tumor cell types and tumor blood vessels, compared to normal tissues. 